\title{
A Hardware Implementation Approach of Eso Based Height Control System of A Sea Skimming Missile
}

\author{
Yashwanth Ranapanga \\ VNR VJIET, Hyderabad, Telangana,India \\ yashwanthranapanga@gmail.com
}

\begin{abstract}
Sea-skimming missiles fly at very low altitude $(5 m-10 m)$ above the mean sea level. An altimeter based height control system is used to keep the missile at the desired helght by measuring the instantaneous height of the missile above the sea level. The altimeter measurement comes with the disturbances which forces the missile to follow the sea waves resulting is a complex trajectory and high energy requirements. An extended state observer based height control system with a proportional derivative controller is used for the real time estimation and rejection of sea wave disturbances to achieve a stable flight path for the missile. Simulations are carried out in both continuous and digital domain to understand the hardware implementation imperatives of the same.
\end{abstract}

Keywords: Sea-skimming missile, Altumeter, Extended State Observer

\section{Introduction}

Missile is a self-propelled weapon hif Jowards a target (enemy weapons). The incorporation of energy sorrce in a misstie to provide the required force for its movement (propulsion), intelligence to go in the correct direction (guidance) and effective manoeuvring (control) are mainly the technologies of guided missiles. The paper is to handle the guidance and control unit the guidance system is to detect whether the missile is flying abbve or below, to the left or right, of the required path. It obtains these deviations or errors and sends) signals to the control system to reduce these errors to zero.

SEA-SKIMMING missiles are anti-ship missiles and they are considered as effective weapon systems against sea and earth surface targets. As the missile flies at lower height above sea surface increase the time to be detected by the enemy ships and making it harder to detect due to radar clutter from the sea. Sea waves effectively act as an active disturbance since the altimeter continuously measures the sea wave elevation [1]. The altimeter makes the missile follow a trajectory parallel to the sea surface, thereby resulting continuous deflections of control surfaces spending unnecessary expense of missile energy and increased chances of the missile ditching into the sea. The unmodeled dynamics and uncertainties in the chosen autopilot model further compound the chances of a mission failure. In [2], an optimal control law is proposed, but the problem of sea waves is not addressed. In [3], a kalman filter is used to estimate the sea waves. Similarly, an algorithm based on the predictive filter approach is proposed in [4] for the design of a height control system by considering the sea-wave Disturbances. Real time sea wave modelling is studied from [5]. In this paper, hardware implementation approach is proposed for height control system based on disturbance estimation. The approach is based on the extended state observer (ESO) [6- 8], wherein the sea-wave disturbances are estimated and cancelled in real time. Initially controller transfer functions are made in continuous domain and converting the transfer function into practical components results into four forms like Foster I and II and Cauer I and II forms. Because of these forms the system may be affected by frequency contents. The controller is then converted into digital domain and simulated. Simulation results show that the system is able to identify 
and reject the disturbances in real time and meet the performance objective in discrete domains as well.

\section{Height Control System}

The basic requirement of a height control system for a sea-skimming missile is that it should fly at a low altitude, above the mean sea level and in rough sea conditions. The height of the sea wave increases causing greater difficulty to the missile. The missile following the sea wave pattern results in enormous wastage in missile energy. The height control system is shown in Figure the major components of basic height control system are lateral autopilot and the radio altimeter.

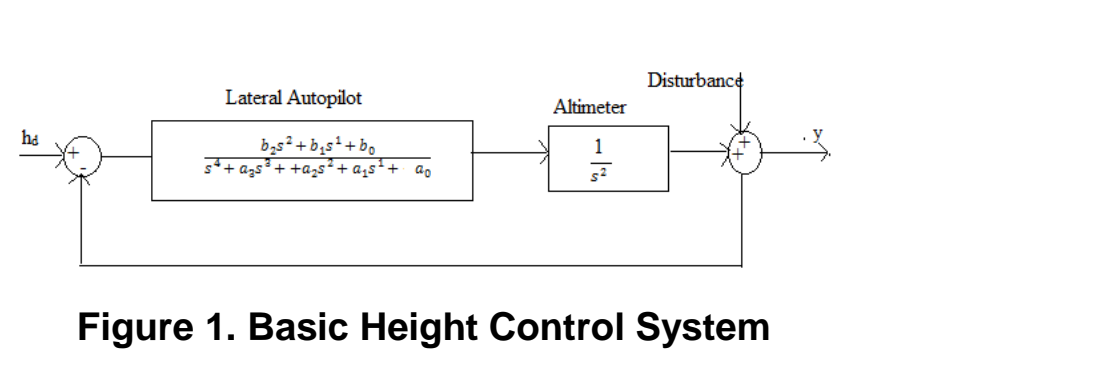

Where $h_{d}, h, v$ and $\hat{h}$ are desired flight path, missile height above sea surface, sea wave disturbance and the height of the missile because of the disturbance.

The task of the control system is 10 -manoeuvre the missile quickly and efficiently making use of these signals. The control system sends signals to the control surfaces of the missile, to change its motion about desired trajectory. The control surfaces are deflected upon receiving the control signalfrom by the servo motor. Servo motors are used inside the guidance and control unit to eonvert linear electrical signal into specific deflection.

An autopilot is aclosed-loop system inside the main guidance subsystem. The function of the autopilot is to stabilize and guide the missile by requesting control surface deflections, which cause the missile body to move either up and down or right and left. A properly designed autopilotprovides a nearly linear response characteristic if changes in these parameters about their nominal design values are small. For autopilots, a number of basic control laws have been designed and analysed over the years [9-13]. In this configuration, an accelerometer provides the main feedback, whereas the rate gyro feedback forms the inner loop of the design and is used to improve the closed-loop damping and stability. The basic configuration of this autopilot is shown in Figure 2. The fin servo is assumed to be a second-order system with $\mathrm{k}_{\mathrm{s}}, \mathrm{w}_{\mathrm{ns}}$, and $\zeta_{\mathrm{s}}$ being the gain, natural/requency, and damping of the servo respectively. The accelerometer improves the stability of the autopilot by provides the main feedback with Ka gain, while the rate gyro with 2 gain $\mathrm{k}_{\mathrm{g}}$ provides the rate feedback to achieve adequate damping in the closed-loop system. The equations that represent the dynamics of a missile are usually highly nonlinear [9].

Where $\mathrm{V}$ is the missile velocity (in meters per second), $\mathrm{w}$ is the component of the velocity along the $\mathrm{Z}$ axis (in meters per second), $\mathrm{q}$ is the pitch rate (in radians per second), and is the control surface deflection (in radians).

The quantities $\mathrm{z}_{\mathrm{w}}, \mathrm{z}_{\mathrm{q}}, \mathrm{z}_{\delta}$, and $\mathrm{m}_{\mathrm{w}}, \mathrm{m}_{\mathrm{q}}, \mathrm{m}_{\delta}$ are the seminondimensional aerodynamic force and moment derivatives, respectively. 


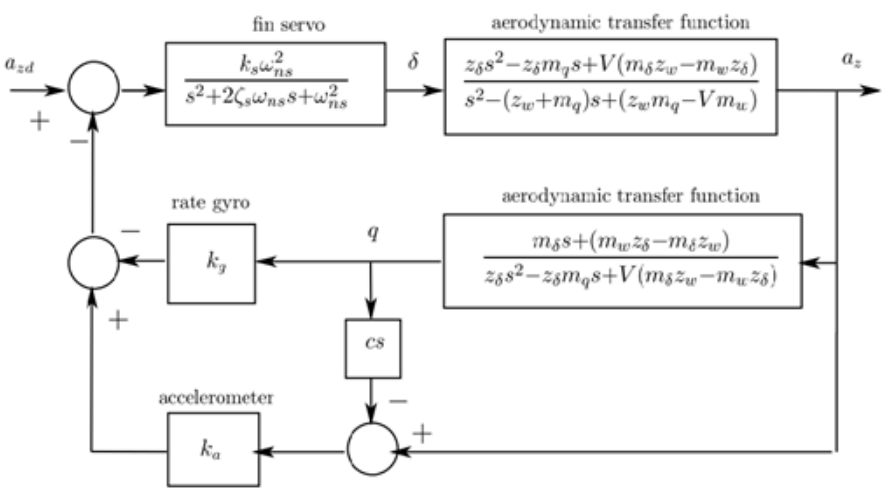

Figure 2. Lateral Autopilot

It can be verified that the autopilot response is well damped and has asteady-state gain nearly equal to unity, with a settling time of approximately $+0.1 \mathrm{~s}$.

\section{Table 1. Autopilot Data}

\begin{tabular}{l|l|l|}
$Z_{w}=-3 \mathrm{~S}^{-1}$ & $Z_{q}=0$ \\
$M_{w}=-1 \mathrm{~m}^{-1} \mathrm{~S}^{-1}$ & $M_{q}=-3 . \mathrm{S}^{-1}$ \\
$K s=0.007$ & getas & $Z_{d}=0.5$
\end{tabular}

and velocity $V=500 \mathrm{~m} / \mathrm{s}$. The output of the per square second) and the (resulting closed-loop transfer function for the autopilot shown in Figure 2 can be written as

$$
\frac{a_{z}(s)}{a_{z d}(s)}=\frac{1}{s_{2}+a_{2} s^{2}++a_{2} s^{2}+a_{1} s^{1}++a_{0}}
$$

where $a_{i}$ and $b_{i}$ are calculated without much difficulty. Using the data in Table.1 one gets the closed-1oop transfer function for the autopilot as

$$
\frac{a_{z}(s)}{a_{z d}(s)}=\frac{-40824 s^{2}-122,472 s^{1}+1.497 * 10^{9}}{s+186 s^{2}++4.67 * 10^{4} s^{2}+3.71 * 10^{6} s^{1}++1.452 * 10^{8}}
$$

The autopilot in the height control system controls the motion of the missile in the pitch plane, so the output of the autopilot is lateral acceleration. As the lateral autopilot forms a part of the height control system, it is necessary to design it properly so that the height control system becomes reliable and satisfies the necessary requirements. It is obyious that the sea-wave disturbance $v$ is essentially equal to $-\mathrm{h}_{\mathrm{s}}$, where $\mathrm{h}_{s}$ is the amplitude of the sea wave with respect to the mean sea level. The presence of double integrators in the loop poses stability problems for the loop thus one needs to design a compensator to make the loop stable.

\subsection{Compensation Network Design}

To achieve stability as well as adequate speed of response for the closed-loop system compensation network has to be designed. In [1], a proportional derivative feedback compensator having a transfer function as along with a lead network in the forward path having a transfer function as are designed to stabilize and obtain a satisfactory transient response for the height control system. To achieve stability and proper transient response the value are:

Lead network $=\frac{K(1+\varangle T 1)}{1+\circlearrowleft T 2} ; \mathrm{K}=5, \mathrm{~T}_{1}=0.8, \mathrm{~T}_{2}=0.01$

PD controller is $1+\mathrm{sT}$; $\mathrm{T}=1, \mathrm{~K}_{0}=1, \mathrm{~K}_{\mathrm{d}}=0.001$. 
The compensated height control system is shown in Figure3. It is straightforward to verify that the open-loop transfer function for the compensated height control system is given as

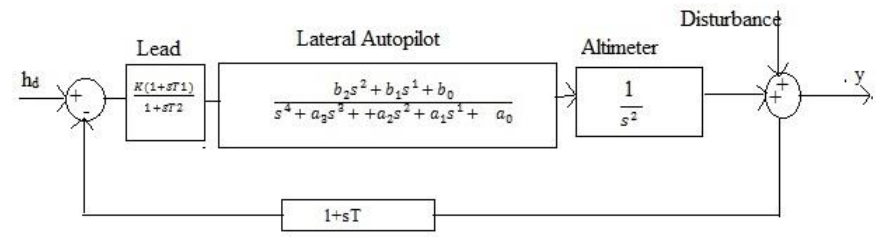

Figure 3. Compensated Height Control System

It is straightforward to verify that the open-loop transfer function for the compensated height control system is given as and it can be easily verified that the closed-loop system is stable with a satisfactory phase margin of $56^{0}$.

$$
G(s)=\frac{K\left(1+s T_{1}\right)\left(b_{2} s^{2}+b_{1} s^{1}+b_{0}\right)(1+s \tau)}{s^{2}\left(1+s T_{2}\right)\left(s^{4}+a_{2} s^{2}++a_{2} s^{2}+a_{1} s^{1}+a_{0}\right)}
$$

\section{Sea Wave Modelling}

An accurate model of the sea-wave disturbance is required to test the performance of the system. The sea-wave pattern varies depending upon the sea state, which is a number ranging from 0 to 9 that describe the general condition of the sea. The sea state is represented with $H_{\text {sig, }}$ the significant wave height given in Table 2, taken from [14]. Previously, to use sea-waves in simulation andysis distinct approaches were used $[1,4$, 15]. The most widely used approach is estimation of sea-states, Pierson-Markowitz spectrum.

The wave amplitude A of wave component I related to the wave spectral density function $\mathrm{s}\left(\mathrm{w}_{\mathrm{i}}\right)$ as

$$
\begin{aligned}
& \mathrm{A}_{\mathrm{i}}^{2}=2 \mathrm{~S}\left(\mathrm{w}_{\mathrm{i}}\right) \Delta \mathrm{w} \\
& \mathrm{S}(\mathrm{w})=A \mathrm{w}^{-5} \exp \left(-\mathrm{Bw}^{-4}\right) \mathrm{m}^{2} \mathrm{~s}
\end{aligned}
$$

where $\mathrm{A}=8.1 \cdot 10^{-3} \mathrm{~g}^{2}$ and $\mathrm{B}=0.74\left(\mathrm{~g} / \mathrm{V}_{\mathrm{w}}\right)^{4}$ The quantity $V w$ is the wind speed above sea surface and $g$ is the gravitational acceleration. Where $w_{i}$ is the wave frequency of wave component $\mathrm{i}$ and $\Delta \mathrm{w}$ is constant difference between successive frequencies. The PiersonMoskowitz spectrum dar be reformulated in terms of $H$ sig, where $H_{\text {sig }}$ is given by

$$
H_{\text {sig }}=0.21\left(\mathrm{~V}^{2}{ }_{w} \mathrm{~g}\right)
$$

the sea state is selected according to the environment desired to simulate. Once the sea state is selected, a value of $\mathrm{Hsig}$ can be chosen from where the spectrum can be caleulated.

Table 2. Sea States and Their Corresponding Wave Elevations

\begin{tabular}{|l|l|l|}
\hline Sea state code & Description of sea & Significant wave height $(\mathrm{m})$ \\
\hline 0 & Calm & 0 \\
\hline 1 & Calm(rippled) & $0-0.1$ \\
\hline 2 & Smooth & $0.1-0.5$ \\
\hline 3 & Slight & $0.5-1.25$ \\
\hline 4 & Moderate & $1.25-2.5$ \\
\hline 5 & Rough & $2.5-4$ \\
\hline 6 & Very rough & $4-6$ \\
\hline 7 & High & $6-9$ \\
\hline 8 & Very high & $9-14$ \\
\hline 9 & Phenomenal & $>14$ \\
\hline
\end{tabular}


The wave elevation at the point of observation, $\mathrm{x}$, is given by

$\zeta(x, t)=\sum_{\mathrm{i}=1}^{N} A_{\mathrm{i}} \cos \left(w_{\mathrm{i}} t-k_{\mathrm{i}} x+\emptyset_{\mathrm{i}}\right)+\sum_{\mathrm{i}=1}^{N} \frac{1}{2} k_{\mathrm{i}} A_{\mathrm{i}}^{2} \cos 2\left(w_{\mathrm{i}} t-k_{\mathrm{i}} x+\emptyset_{\mathrm{i}}\right)$
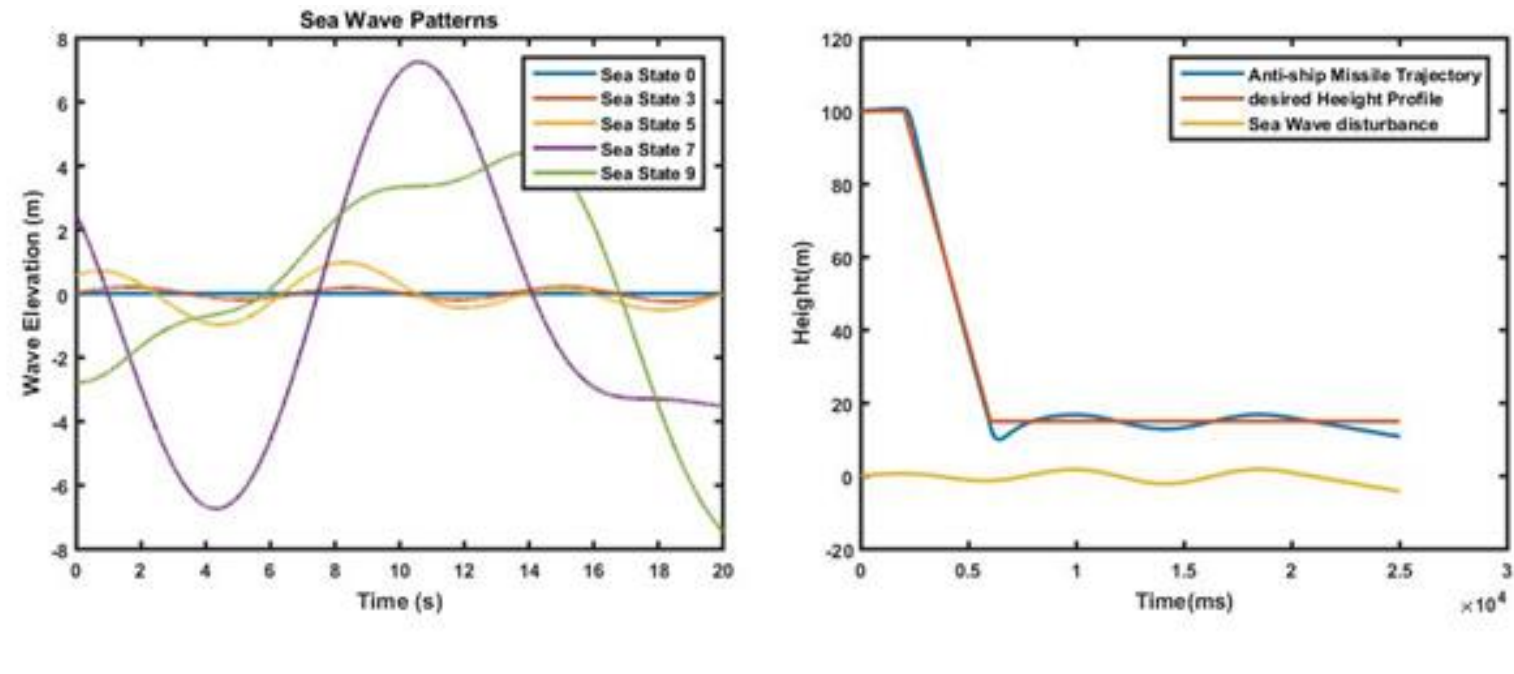

Figure 4. Sea Waves with Different Statesand the Response of the Compensated Height Control System tor Sea State 5

\section{State Observers and Analysis on Extended State Observer}

There are many situations in the modern technology in which it is necessary to 1 estimate the state of a dynamic system using only the measured input and output data of the system. A state observer nay be understood as an auxiliary system that is employed to estimate a non-observed state. The observer is a very useful tool for receiving information of the variables of a system that are unknown. For this reason, it is usually employed in control systems where knowledge of the plant system is necessary from an incomplete observation. The use of state observers is related to estimating, controlling and also deteeting and identifying failures in dynamical systems.

\subsection{Extended State Observer}

Nonlinear systems have many types of uncertainties, such as imperfections of mechanisms, unknown nonlinearities and parameters, which make impossible to obtain an exact function of disturbance. The ESO is an observer that can estimate the effects of uncertainties and disturbances along with the states of the system and it is able reject the disturbance. The ESO observes all factors affecting the plant, including the nonlinearities, uncertainties, effects of unmodeled dynamics, and external disturbances, as a total disturbance. In [6] a comparison study of the performances and characteristics of three advanced state observers, namely, high gain observer, ESO, and sliding mode observer is presented, and it is shown that, overall, the ESO is dealing excellently with the uncertainties, disturbances and sensor noise.

Consider a single input single output system

$$
\ddot{y}=a(y, y, w)+b u
$$

where $\mathrm{a}($.$) represents the system, \mathrm{w}(\mathrm{t})$ is an unknown disturbance, $\mathrm{u}$ is the control signal and $y$ is the measured output. Let $b=b o+\Delta b$, where bo is the best available estimate of $b$, and $\Delta \mathrm{b}$ is its associated uncertainties. Defining the uncertainty to be determined as $\mathrm{d}$ $\triangleq \mathrm{a}()+.\Delta$ bu and designating it as an extended state $\mathrm{x} 3$, the dynamics of Eq. (11) can be written in a state-space form as

$$
\dot{x}_{1}=\mathrm{x}_{2} \quad \dot{x}_{2}=\mathrm{x}_{3}+\mathrm{b}_{0} \mathrm{u} \quad \dot{x}_{3}=\mathrm{f} \quad \mathrm{y}=\mathrm{x}_{1}
$$


Iwhere $f$ is the rate of change of the uncertainty; that is $f=d$ an unknown bounded function. By making $d$ as a state, it is possible to estimate it by using a state estimator. Consider a nonlinear observer of the form

$$
\begin{aligned}
& \dot{\hat{x}}_{1}=\hat{x}_{2}+\beta_{1} \mathrm{~g}_{1}(\mathrm{e}) \quad \dot{\dot{x}}_{2}=\hat{x}_{3}+\beta_{2} \mathrm{~g}_{2}(\mathrm{e})+\mathrm{b}_{0} \mathrm{u} \quad \dot{\dot{x}_{3}}=\beta_{3} \mathrm{~g}_{3}(\mathrm{e}) \\
& \mathrm{y}=\hat{x}
\end{aligned}
$$

where $e=y-\hat{y}=x_{1}-\hat{x}_{1}$, and $\hat{x}_{3}$ is an estimate of the uncertainty. The quantities $\beta i$ are the observer gains, forms matrix $\mathrm{H}$.

Rewriting the dynamics of the ESO given by eq(17).

$$
\dot{x}_{e}=A_{e} x_{e}+B_{e} u+E f ; y=C_{e} x_{e}
$$

where $\mathrm{x}_{\mathrm{e}}$ is the extended state vector i.e.,

$$
\begin{aligned}
& \mathrm{x}_{\mathrm{e}}=\left[\begin{array}{lll}
\mathrm{x}_{1} & \mathrm{x}_{2} & \mathrm{x}_{3}
\end{array}\right]^{\mathrm{T}} \\
& \mathrm{Ae}=\left\{\begin{array}{lll}
0 & 1 & 0 \\
0 & 0 & 1 \\
0 & 0 & 0
\end{array}\right\} \quad \mathrm{Be}=\left[\begin{array}{c}
0 \\
b 0 \\
0
\end{array}\right] \quad \mathrm{Ce}=\left[\begin{array}{lll}
1 & 0 & 0
\end{array}\right] \text {; } \\
& \mathrm{E}=\left[\begin{array}{lll}
0 & 0 & 1
\end{array}\right]^{\mathrm{T}}
\end{aligned}
$$

where variable $x 3=f(x 1, x 2, w(x, t))$ represents an extended state. It should be pointed out that, both $f$ and its derivative $h=f$ are unknown. However, by making $f$ as a state, it is now possible to estimate it by using a state observer. Form for the state observer:

$$
\dot{\vec{x}}=\hat{A} x+B u+H G(e)
$$

Here, $\hat{x}=\left\{\hat{x}_{1} \hat{x}_{2} \hat{x}_{3}\right\} T$ are estimated values of vector $\hat{x}_{0}$ Notice that $\hat{x}$ represents an estimative of $f\left(x_{1}, x_{2}, w(x, t)\right)$ and $e=x_{1}-\alpha_{1}$ represents the error associated with the estimation. If the initial state of the process under obserdation known precisely (i.e., $\mathrm{x}_{0}=\hat{x}$ ) then the estimation error is zero thereafter. Due to the possibility of the occurrence of disturbances, however, the trute state $\mathrm{x}$ may depart from the solution to during the course of operation of the observer. Hence knowledge of the initial state $\mathrm{x}\left(t_{0}\right)$ does not eliminate the need for an observer in practican situations. Since the matrices A, B, and C are defined by the plant, the only choice in the design of the observer is in the selection of the gain matrix $\mathrm{H}$. Selecting the values of gain matrix $\mathrm{H}$ plays turning role in the designing of the observer, the gain values are chbsen properly, such that the error converges to zero and $\mathrm{H}$ can be obtained using known method such as pole placement method. In this equation, the following yectors are used:

$\mathrm{H}=\left[\begin{array}{lll}\beta 1 & \beta 2 & \beta 3\end{array}\right]^{\mathrm{T}}$

$$
\mathrm{G}(\mathrm{e})=[\mathrm{g} 1(\mathrm{e}) \mathrm{g} 2(\mathrm{e}) \mathrm{g} 3(\mathrm{e})]
$$

if $g i(e)=e$, the ESO takes the form of the conventional Luenberger observer and is designatedas the linear ESO. The observer gains can be chosen systematically through pole placement technique. Also, the LESO is easy from a hardware implementation point of view, as is shown in [24]. The gain of the observer is chosen in order to obtain a good estimative for the system variables. Therefore, function $g i$ is defined as a modified exponential

gain function:

$$
g i(e, \alpha i, \delta)=\left\{\begin{array}{c}
|e|^{\wedge}(\alpha i) \operatorname{sign}(e),|e|>\delta \\
e / \delta^{\wedge}(1-\alpha i),|e| \leq \delta
\end{array}\right.
$$

The parameter $\delta$ is a small number used to limit the gain in the neighbourhood of the origin. This procedure prevents excessive gain when the error is small avoiding high frequency chattering. Therefore, when $0<\alpha_{i}<1, g_{i}$ yields high gain for small errors, $|e| \leq$ $\delta$. When $\alpha_{i}<0$, a reduction in observed error occurs. Usually, the first trial considers a linear gain $(\alpha i=1, i=1,2,3), g_{i}(e, \alpha i, \delta)=e(i=1,2,3)$. Under this assumption, the pole placement method can be used for the initial design of this observer. Nonlinearities can be added in order to improve the performance of the observer. The nonlinear gain functions introduce higher complexity in the estimation algorithm. The stability of the observer is 
assured from an appropriate choice of gain values $\beta i$. The stability of a linear extended state observer can be analysed by subtracting the observer equation (7) from system equation (6). This procedure gives an equation for the error dynamics:

$e^{\cdot}=A_{e} e+E f$

where

$$
A e=A-H C=\left[\begin{array}{lll}
-\beta 1 & 1 & 0 \\
-\beta 2 & 0 & 1 \\
-\beta 3 & 0 & 0
\end{array}\right]
$$

where $\mathrm{e}=\mathrm{x}-\mathrm{x}^{\wedge}$ state estimation vector. Since the pair $\left(A_{e}, C_{e}\right)$ is observable, one can compute the observer gain $L$, and so the ESO gains $g_{i}$, such that the observer error dynamics are stable and have the desired transient behaviour. As the eigenvalues of the observer error dynamics given by Eq. (19) are placed at the desired locations, it can be stated that, under the assumption of boundedness of $f$, bounded-input-bounded-oxtput stability for the linear system of Eq. (19) is assured. A special case arises when $f=0$, i.e., when the rate of change of uncertainty $d$ is zero. In this circumstance, the errors will go to zero asymptotically i.e,

$$
\lim _{t \rightarrow \infty} e(t)=0 \text {. }
$$

Some of the ESO applications are found in [17/, 18$],[19]$ and [201

\section{B. Pole Placement Technique}

The stability of the error dynamics is associated with the roots of the characteristic polynomial of $A_{e}$, since $f$ is bounded:

$$
\lambda(s)=s^{3}+\beta_{1} s^{2}+\beta_{2} s+\beta_{3}=0
$$

In order to tune the linear ESO, the roots must be all in the open left-half. Pole placement technique gives a condition under which is possible to place system poles (eigen values of the characteristic equationsin the desired locations. [7] defines the woparameterization where allobserver eigen values must be equal to - $\omega$ o i.e, the poles should be in the left half of the s-plane

Therefore, $\left.H_{A} \in \beta 1 \quad \beta 2 \beta 3\right]^{T}$ becomes a function of $\omega o$, which is denoted as the bandwidth of the observer. Then.

$$
\lambda(s)=s^{3}+\beta_{1} s^{2}+\beta_{3} s+\beta_{3}=\left(s+\omega_{0}\right)^{3}=0
$$

Solving this equation, one obtains the following relationship between $\beta i$ and $\omega o$ :

$$
\beta_{1}=3 \omega_{0}, \beta_{2}=3 \omega^{2}, \beta_{3}=\omega^{3}{ }_{\mathrm{o}}
$$

\section{Mathematical Modelling of the Height Control System Implementing Extended State Observer and the Simulation}

The ESO is now introduced into the height control loop. To this end, it is assumed that the plant is given by a double integrator in the forward loop. This assumption is valid, since the autopilot has a steady-state gain of unity and a substantially smaller settling time in comparison with the closed-loop height control system. The representation for the plant in this case is

$$
\left[\begin{array}{l}
\mathrm{x}^{0} 1 \\
\mathrm{x}_{2}
\end{array}\right]=\left[\begin{array}{ll}
0 & 1 \\
0 & 0
\end{array}\right]\left[\begin{array}{l}
\mathrm{x} 1 \\
\mathrm{x} 2
\end{array}\right]+\left[\begin{array}{l}
0 \\
1
\end{array}\right] ; \mathrm{y}=\left[\begin{array}{ll}
1 & 0
\end{array}\right]\left[\begin{array}{l}
\mathrm{x} 1 \\
\mathrm{x} 2
\end{array}\right]+\mathrm{v}
$$

where $v$ is the sea-wave disturbance. Since the ESO can estimate unknown plant dynamics, including input disturbance, and not the measurement noise, it is necessary to rewrite Eq. (20) in a suitable form. The equivalent input disturbance signal is $v_{0}=s^{2} v$ and rewriting Eq. (20) as

$$
\left[\begin{array}{l}
x^{x} 1 \\
x_{2}
\end{array}\right]=\left[\begin{array}{ll}
0 & 1 \\
0 & 0
\end{array}\right]\left[\begin{array}{l}
x 1 \\
x 2
\end{array}\right]+\left[\begin{array}{l}
0 \\
1
\end{array}\right] u+\left[\begin{array}{l}
0 \\
1
\end{array}\right] v^{r} \quad y=\left[\begin{array}{ll}
1 & 0
\end{array}\right]\left[\begin{array}{l}
x 1 \\
x 2
\end{array}\right]+v
$$


Nominating $v^{\prime}$ as an extended state $x_{3}$, the resulting extended-order system is in the same form as given in Eq. (15) with $b o=1$. Eq. (17) can then be designed to obtain the estimation of input disturbance $v_{0}$. As $\hat{x}_{3}$ is an estimate of $v_{0}$, the actual sea wave disturbance $v$ is obtained by double integration of $\hat{x}_{3}$ and is subtracted from the measured height to obtain the height above mean level. The functional block diagram of the ESObased height control system is shown in Figure

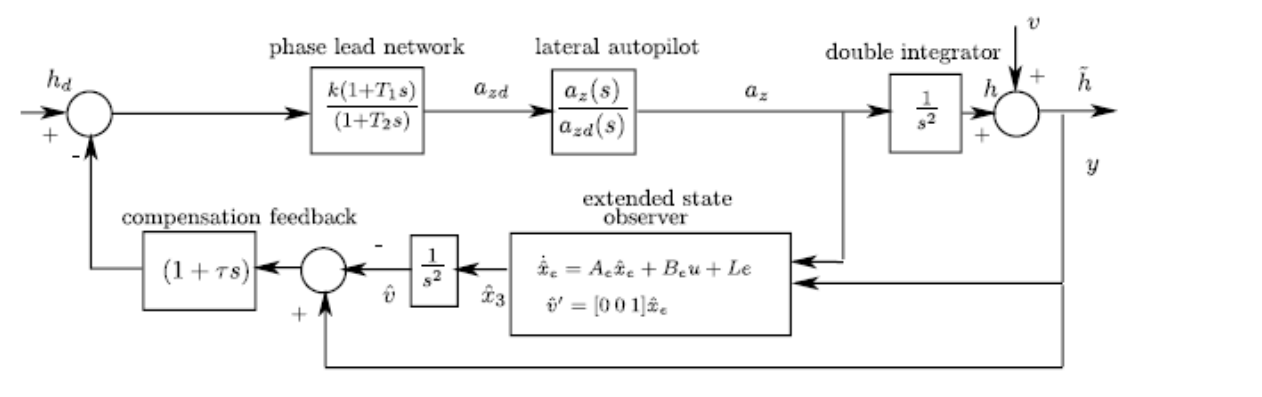

Figure5. Block Diagram of ESO based Height Control System

\section{ESO Analysis}

As discussed earlier, the ESO is used to estimate the sea-wave disturbance. The estimated sea-wave disturbance, denoted as $\hat{v}(\mathrm{~s})$ in Figure, is subtracted from the measured height $y(s)$ in order to reject the disturbance. From the ESO dynamics shown in the figure, the mathematical calculafions are inspired from [22] and the quantity $\hat{x}_{3}(\mathrm{~s})$ is given by

$$
\hat{\mathrm{x}}_{3}=-\frac{\beta \mathrm{a}}{\mathrm{a}} \mathrm{a}_{\mathrm{z}}(\mathrm{s})+\frac{\beta \mathrm{a}}{\mathrm{a}} \mathrm{s}^{2} \mathrm{y}(\mathrm{s})
$$

where $\Delta \triangleq s^{3}+\beta_{1} s^{2}+\beta s s+\beta_{3}$ and $\beta$ are the observer gains. As seen in the figure, the estimated sea-wave disturbance $\hat{v}(s)$ is given by

$$
\hat{v}(\mathrm{~s})=1 / \mathrm{s}^{2}, \mathrm{x}_{3}(\mathrm{~s})
$$

Substituting Eq. (29) into EC (28) leads to

$$
\hat{\mathrm{v}}(\mathrm{s})=1 / \mathrm{s}^{2}\left[-\frac{\beta a}{a} \mathrm{a}_{\mathrm{z}}(\mathrm{s})+\left[\mathrm{Bz} \mathrm{s}^{2} \mathrm{y}(\mathrm{s})\right]\right.
$$

Since $y(s)=v(s)+1 / s^{2} a_{2}(s)$, substituting in Eq. (30) gives

$$
\hat{v}(\mathrm{~s})=\beta_{3} /\left(s^{3}+\beta_{1} s^{2}+\beta_{2} s+\beta_{3}\right)
$$

Thus, the estinnated sea-wave disturbance can be considered as a signal acting on the height control. An equivalent functional block diagram of the ESO-based height control system explicitly bringing out the dynamics of the disturbance observer is shown in Figure As can be seen from the figure, the loop transfer function for the height control loop is given by

$$
L(s)=\frac{K\left(1+\Delta T_{1}\right)\left(a_{z} g s(1+\Delta T)\right.}{s^{2}\left(1+s T_{2}\right) a_{z d^{g}}}
$$

The stability of the ESO-based height control system is decided by the compensated height control loop, however, its performance is governed by the ESO estimation dynamics. It is desirable that the ESO estimates the sea-wave disturbance as fast and as accurately as possible to achieve desired performance. The gains $\beta_{i}$, and so the ESO observer gain vector $\mathrm{H}$ as required in Eq. (21), are obtained by using a pole placement technique. To this end, all poles of the observer dynamics are placed at -2000 to achieve fast convergence of the disturbance estimation, and the corresponding gains are found as $\beta_{1}=6000, \beta_{2}=1.2 * 10^{7}$ and $\beta_{3}=8 * 10^{9}$. 


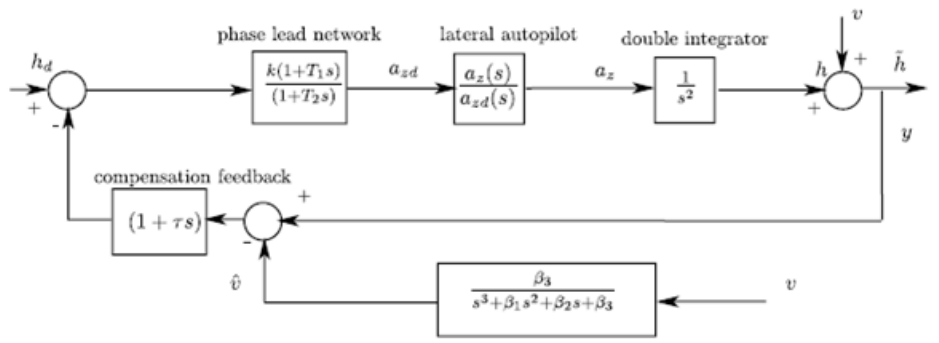

Figure 6. Equivalent Block Diagram of ESO Based Height Control System

The resulting open-loop bode plot is found and it can be observed that the closed-loop system is stable with a satisfactory phase margin of 56.60. The closed-loop transfer function gives the closed-loop poles as $-1.22,-1: 29,-12: 6+j 55.5,-41.5$, and $-63.4+j 177$. From the above poles, it can be observed that the slow poles are on a ear axis, giving effective damping of unity, thus, the overshoot is expected to be smalh, as desired in the height control problem. To show the height simulation result, it is necessary to generate a desired height profile which acts as input set height profile. For the present case, the reference height profile is generated as follows:

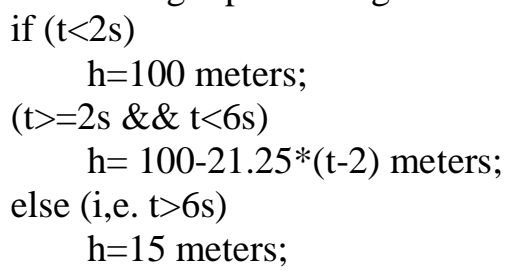

First, simulations are carried olut for the compensated height control system without the ESO shown in Figure 5 and the ESO-based neight control system shown in Figure 6 for the sea states of 0, 5, 7 and 9 . In the simulations of the ESO-based height control system, the disturbance rejection is commenced after the initial disturbance estimation transients are over. The simulation results are presented in Figure
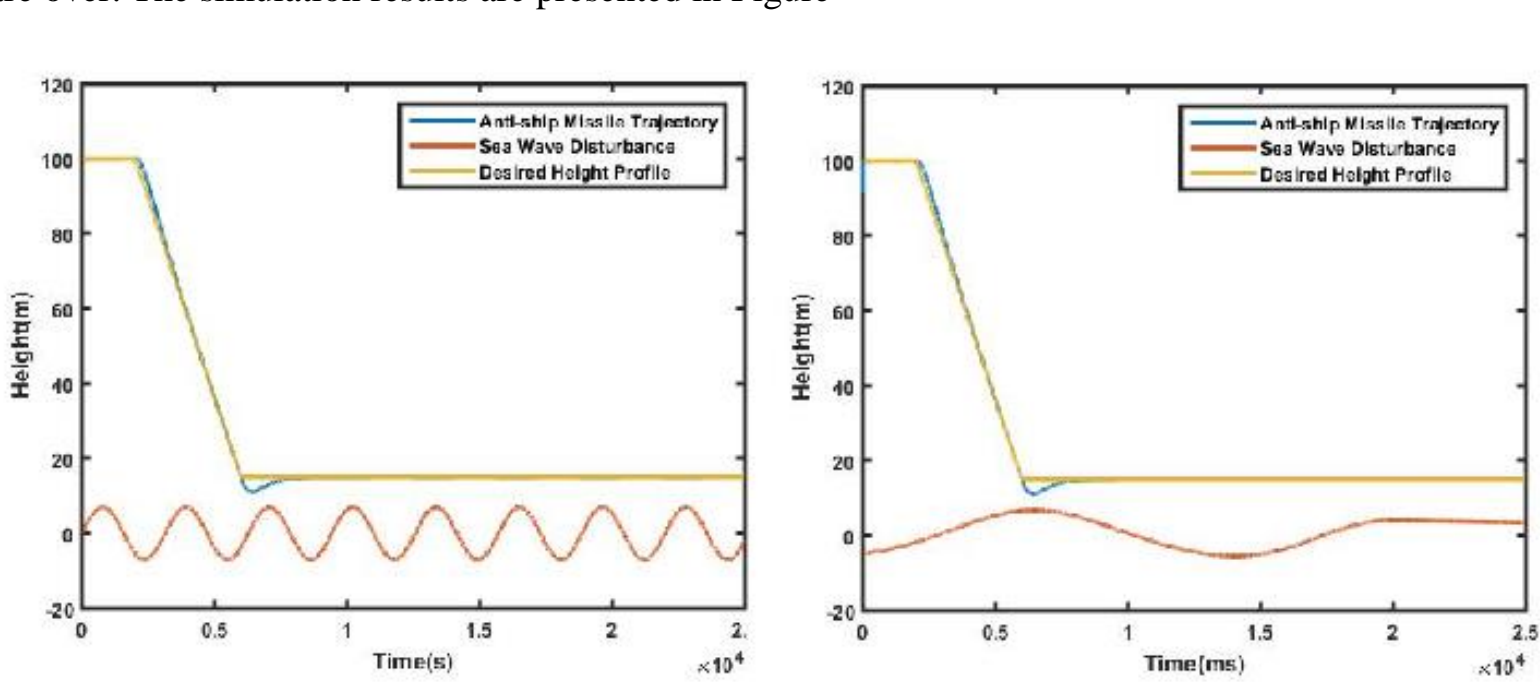

Figure 7. Simulation with A Conventional Sine Wave and With Seas State 7 (Implementing ESO) 


\section{Digital Domain Transformation}

In the previous section continuous domain transfer functions are obtained. From the transfer functions with the aid of network synthesis techniques like cauer and foster forms the elements of the transfer function can be obtained. The elements in the analogue domain are generally resistance, capacitance and inductance. From the calculations it is observed that the values of the energy storage elements are in the few faraday's and in few henry's. As the value of these elements increases its size increases automatically, therefore overall size of the missile increases drastically, this is not recommended to increase the size of the missile. Most importantly the two $\mathrm{C}$ and $\mathrm{L}$ should not present in the same circuit because of the frequency problems. When $\mathrm{C}$ and $\mathrm{L}$ are present in a network resonance problem may arise and the bandwidth limitation will als $\varnothing$ apply, because of these reasons it is not preferable to implement the ESO hardware in the continuous domain. On the other hand the cost of the system will be sky height.

More convincing solution for this problem is to convert the continuous dormain system into digital domain, so that all the problems specified above will be resolved with this transformation, can be implemented practically with the use of micro controllers. The basic idea for using microcontrollers is very simple that $\mathrm{c}++$ code can be implemented directly by the use of microcontrollers. The software used for the simulation study has the capability to convert analogue function into digital, later the digital response can be converted into c/c++ codes. By executing the $\mathrm{e} / \mathrm{c} *+$ code with microcontrollers completes the task of implementation practically.

The continuous control system is then converted jeto digital domain and simulated the response similar to the continuous domain response. The discrete domain response is shown in the Figure

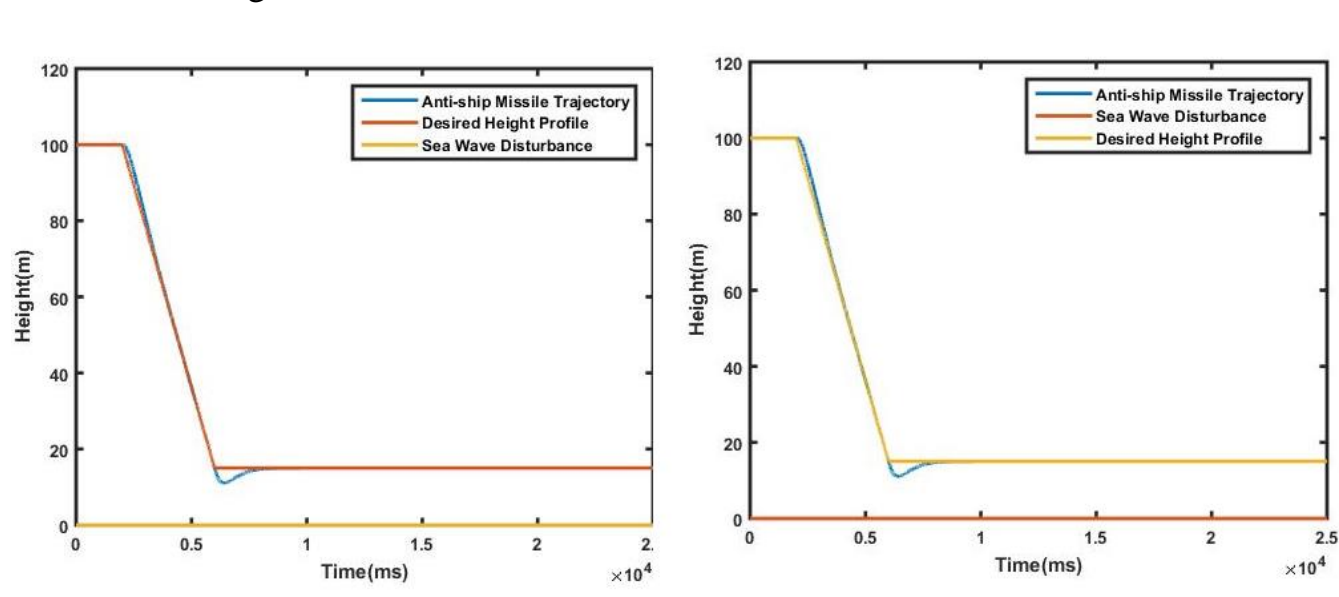

A) Sinulation Results with Sea State 0, Continuous and Discontinuous Respectively.
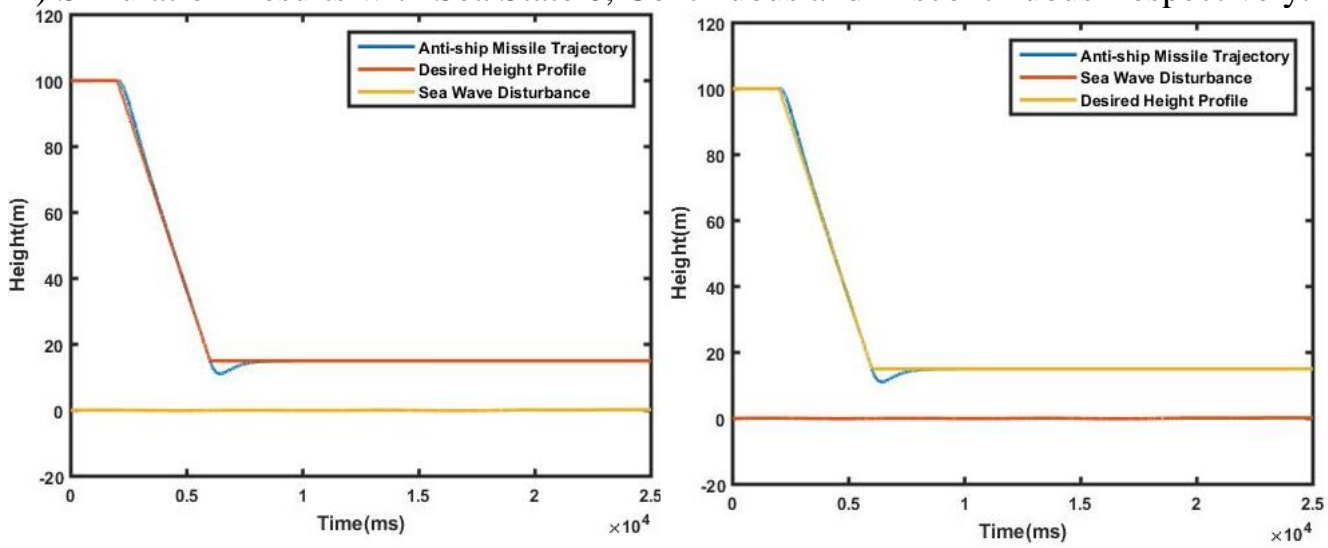

B) Simulation Results with Sea State 3, Discontinuous and Continuous Respectively. 

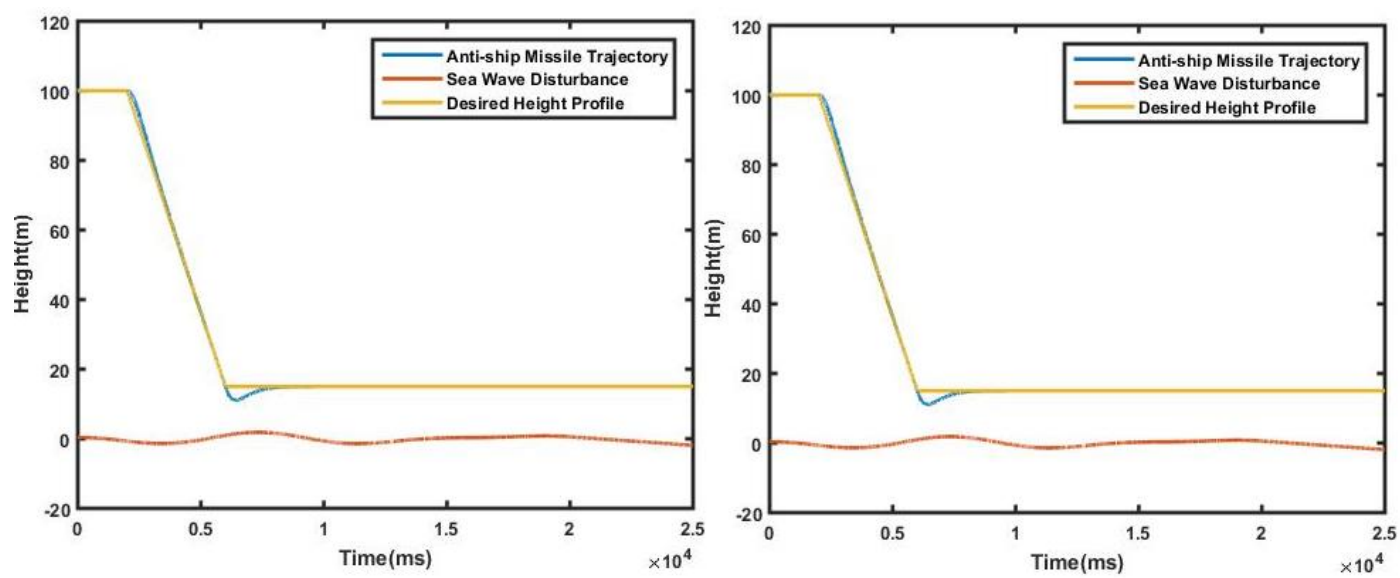

C) Simulation Results with Sea State 5, Continuous and Discontinuous Respectively.
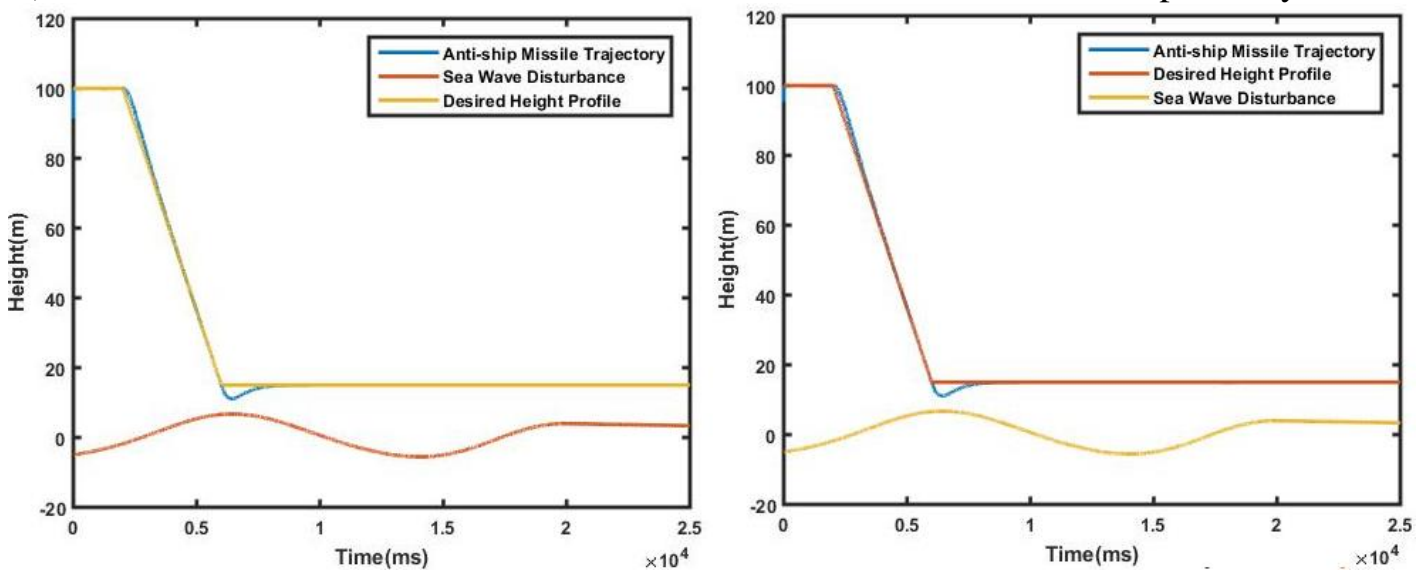

D) Simulation Results with Sea State) 7 , Continuous and Discontinuous Respectively.
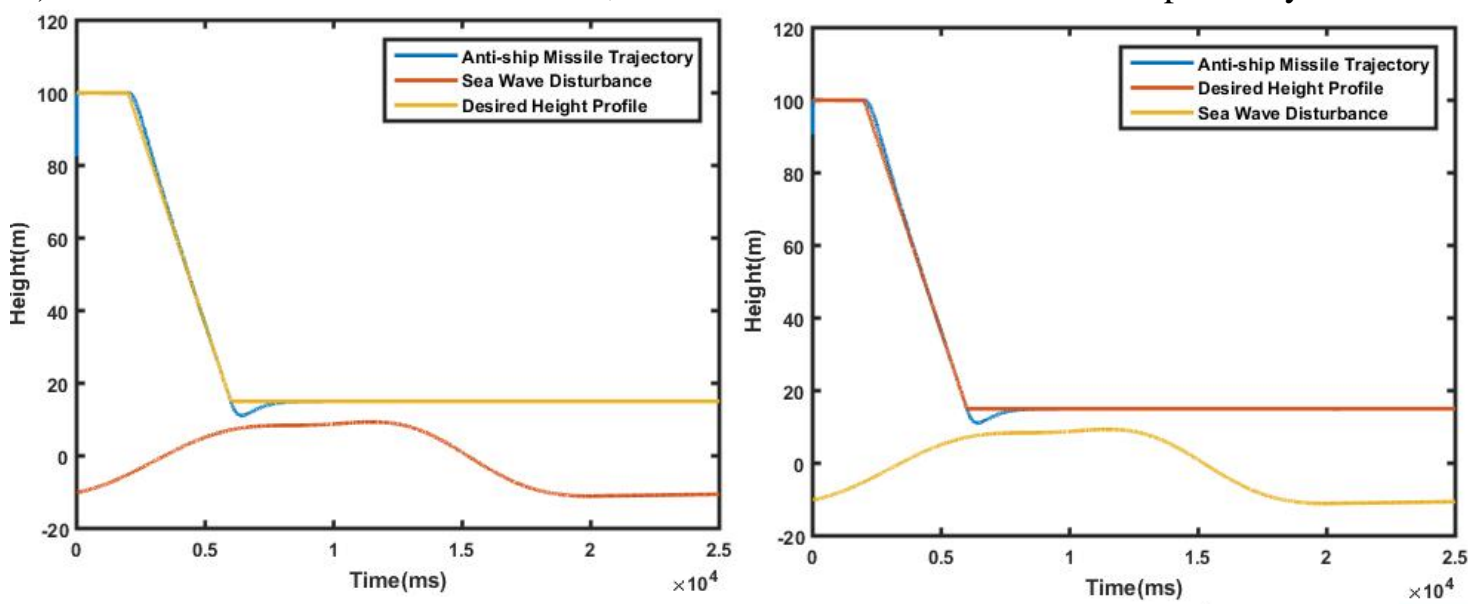

E) Simulation Results with Sea State 9, Continuous and Discontinuous Respectively.

Figure 8. Comparison of Missile Trajectory with Different Sea States in Both Continuous and Discrete Domains

\section{Conclusion}

An ESO-based approach is presented for the height control system design for a seaskimming missile. The sea-wave disturbance acting on the control system is estimated by the ESO as an extended state of the system. The disturbance is then cancelled using the disturbance rejection approach. The simulation results show that the proposed height 
control system performs satisfactorily under all sea conditions and meets the objective of the design for a sea-skimming missile. Performance comparison of the both the domains is analysed and few points were dealt regarding hardware implementation approach. Hardware implementation can be done using micro controllers which execute the c/c++ code generated from the digital response of the missile control system.

\section{References}

[1] R. Sutton and C. L.Windley, "Stabilization and Simulation of the Height Control System of a Sea Skimming Missile", Journal of Naval Science, vol. 11, no. 4, (1985), pp. 248-256.

[2] J. R. Dowdle, "An Optimal Guidance Law for Supersonic Sea Skimming”, AIAA Guidance, Navigation, and Control Conference, AIAA Paper 1985-1866, (1985), pp. 165-169.

[3] J. R. Dowdle and P. Kim, "Bandwidth Requirements for Sea Skimming Guidance", AIAA Guidance, Navigation, and Control Conference, AIAA Paper 1985-1867, (1985), pp. 170-175.

[4] S. E. Talole and S. B. Phadke, "Height Control System for Sea Skimming Missile Using Predictive Filter", Journal of Guidance, Control, and Dynamics, vol. 25, no. 5, (2002), pp. 989-992.

[5] T. I. Fossen, "Guidance and Control of Ocean Vehicles", Wiley,New York, (1999), pp. $57-70$.

[6] W. Wang and Z. Gao, "A Comparison Study of Advanced State Observer Design Techniques", Proceedings of the American Control Conference, Denver, CO, LEEE Publ., Piscatayay, NJ, (2003),pp. 4754-4759.

[7] Z. Gao, "Scaling and Bandwidth-Parametrization based Controller Tuning", Proceedings of the American Control Conference, Denver, CO, IEEE Publ., Piseataway, NJ, (2003), pp. 4989-4996.

[8] D. Yoo, S. S.-T. Yau and Z. Gao, "Optimal Fast Tracking Observer Bandwidth of the Linear Extended State Observer", International Journal of Control vol. 80, no. d, (2007), pp. 102-111.

[9] P. Garnell, "Guided Weapon Control Systems", Pergamon, 2nd ed., Oxford, England, U.K., (1980).

[10] P. H. Reisenthel, "Development of a Nonlinear Indicial Model using Response Functions Generated by a Neural Network", AIAA Paper 1997-0337.(1997).

[11]O. M. Faltinsen, "Sea Loads on Ships and Offshore, Structures", Cambridge Univ. Press, New York, (1990), pp. 13-34.

[12] D. Luenberger, "observers for multivariable systems", IEEE Trans. on automatic control vol. AC11,pp,190-197.

[13] J. Han, "A class of extended state observers for uncertain systems", Contr. Decis. Vol.10, (1995), 8588.

[14] W. Wang and Z.Gao "Á Comparison Study of Advanced State Observer Design Techniques", Proceedings of the American Control Conference, Denver, CO, IEEE Publ., Piscataway, NJ, (2003), pp. 4754-4759.

[15] S. E. Talole, J. P. Kolhe and S. B. Phadke, "Extended-State-Observer-Based Control of Flexible-Joint System with Experimental Validation", IEEE Transactions on Industrial Electronics, vol. 57, no. 4, (2010), pp. 1411-1419.

[16] F. Heitor and I. Pereira Pinto, "State Space Reconstruction Using Extended State Observers To Control Chaos In A Noninnear Pendulum", International Journal Of Bifurcation And Chaos, vol. 15, no. 12, (2005), pp, $4051-4063$.

[17] J. Su, W. Qiu, H. Ma and P.-Y. Woo, "Calibration-Free Robotic Eye-Han Coordination Based on an Auto Distubance Rejection Controller”, IEEE Transactions on Robotics, vol. 20, no. 5, (2004), pp. 899907.

[18] Y. X. Su, C. H. Zheng and B. Y. Duan, "Automatic Disturbances Rejection Controller for Precise Motion Control of Permanent-Magnet Synchronous Motors", IEEE Transactions on Industrial Electronics, vol. 52, no. 3, (2005), pp. 814-823.

[19] Y. X. Su, B. Y. Duan, C. H. Zheng, Y. F. Zhang, G. D. Chen and J. W. Mi, "Disturbance-Rejection High-Precision Motion Control of a Stewart Platform", IEEE Transactions on Control Systems Technology, vol. 12, no. 3, (2004), pp. 364-374.

[20] R. Zhang and C. Tong, "Torsional Vibration Control of the Main Drive System of a Rolling Mill Based on an Extended State Observer and Linear Quadratic Control”, Journal of Vibration and Control, vol. 12, no. 3, (2006), pp. 313-327.

[21] S. E. Talole, J. P.Kolhe and S. B. Phadke, "Extended-State-Observer- Based Control of Flexible-Joint System with Experimental Validation", IEEE Transactions on Industrial Electronics, vol. 57, no. 4, (2010), pp. 1411-1419.

[22] Priyamvada, "Robust Height Control System Design For Sea-Skimming Missiles", Journal Of Guidance, Control, And Dynamics, vol. 34, no. 6, (2011),pp,1746-1756. 


\section{Author}

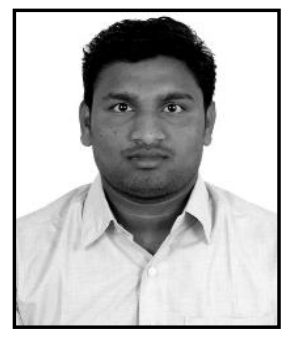

Yashwanth Ranapanga, $\mathrm{He}$ completed his Master of Technology in Electronics and Instrumentation Engineering. VNR Vignana Jyothi College of Engineering \& Technology, Hyderabad, Telangana, India. He completed his Bachelor of Technology from Mahatma Gandhi Institute of Technology, Hyderabad, Telangana, India in the field of Electrical and Electronics Engineering. His research interests are in the areas of Industrial Automation and Advanced Control Systems.

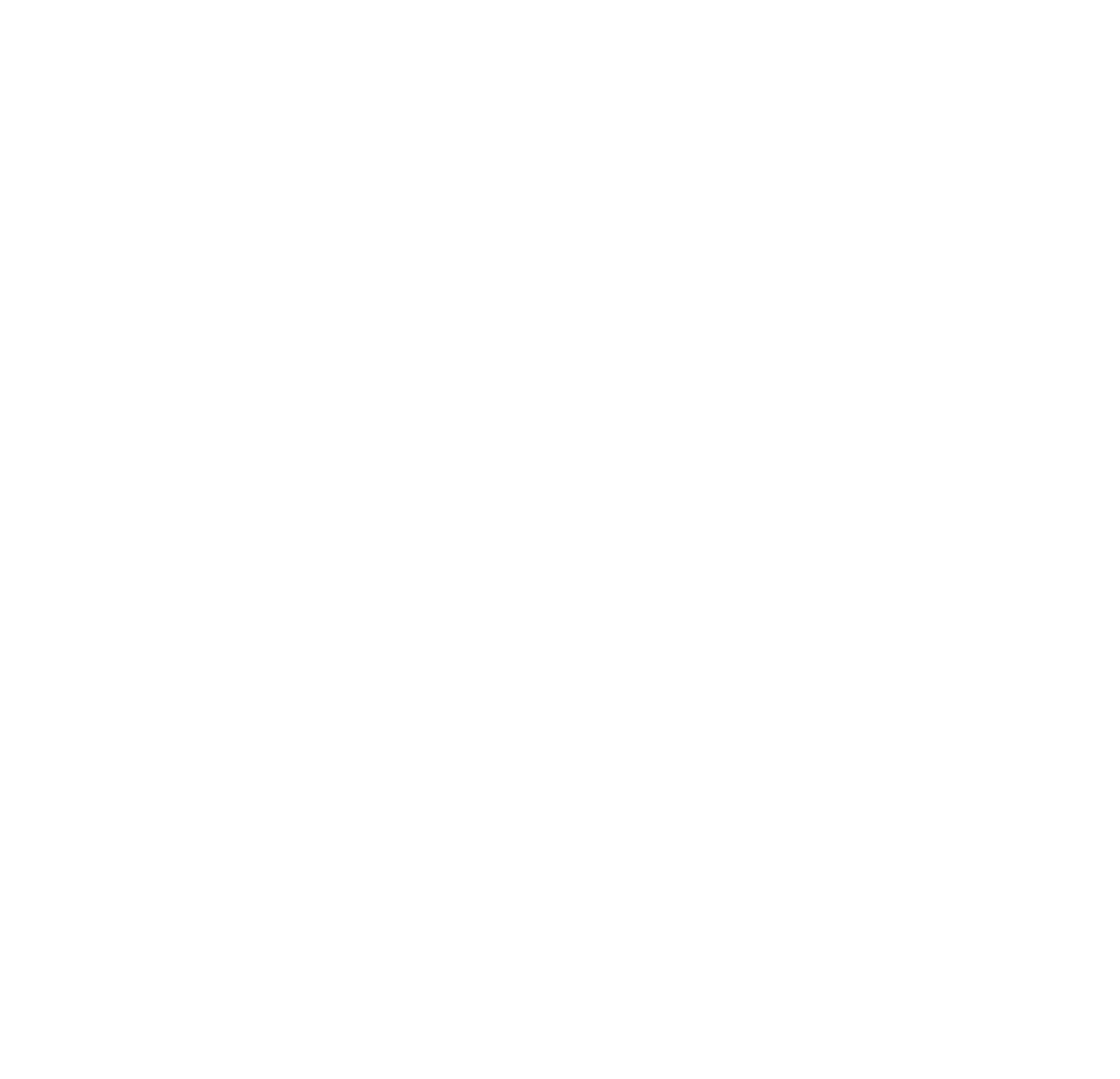


International Journal of Control and Automation

Vol. 9, No. 8 (2016)

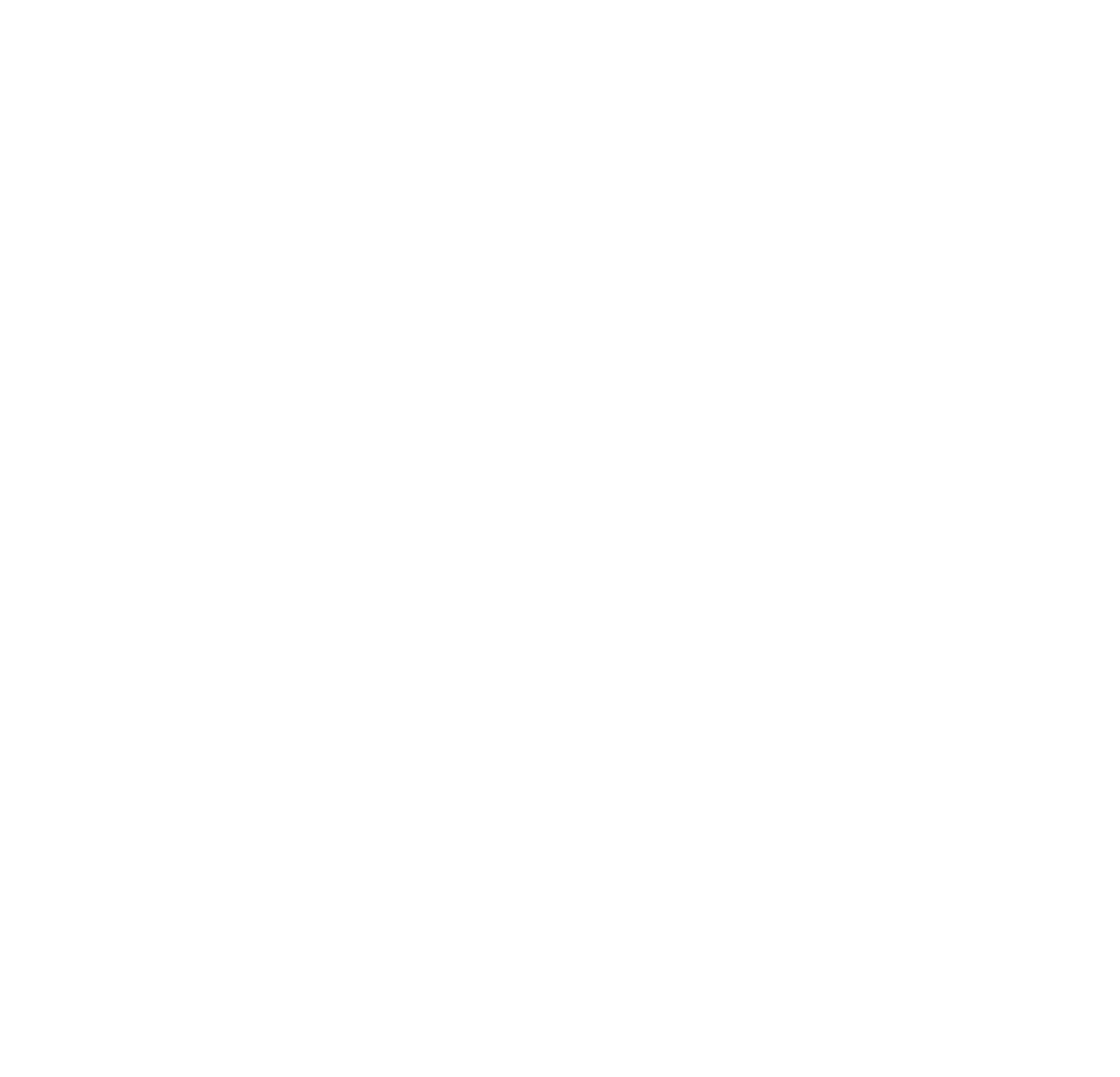

